\title{
Sounds of sickness: Can people identify infectious disease using sounds of coughs and sneezes?
}

\author{
Nicholas M. Michalak ${ }^{1}$, Oliver Sng ${ }^{2}$, Iris M. Wang ${ }^{1}$, \& Joshua M. Ackerman ${ }^{1}$ \\ ${ }^{1}$ University of Michigan \\ ${ }^{2}$ University of California-Irvine
}

\begin{abstract}
In press at Proceedings of the Royal Society B: Biological Sciences
Cough, cough. Is that person sick, or do they just have a throat tickle? A growing body of research suggests pathogen threats shape key aspects of human sociality. However, less research has investigated specific processes involved in pathogen threat detection. Here, we examine whether perceivers can accurately detect pathogen threats using an understudied sensory modality - sound. Participants in four studies judged whether cough and sneeze sounds were produced by people infected with a communicable disease or not. We found no evidence that participants could accurately identify the origins of these sounds. Instead, the more disgusting they perceived a sound to be, the more likely they were to judge that it came from an infected person (regardless of whether it did). Thus, unlike research indicating perceivers can accurately diagnose infection using other sensory modalities (e.g., sight, smell), we find people overperceive pathogen threat in subjectively disgusting sounds.
\end{abstract}

Keywords: behavioral immune system, pathogen detection, auditory perception, evolutionary psychology, accuracy

Word count: 5477

Around $15 \%$ of human mortality around the world is caused by infectious and parasitic diseases (Organization, 2018). This is relatively good news, as infectious diseases have likely been one of the greatest selection pressures on our species' evolution (Karlsson, Kwiatkowski, \& Sabeti, 2014). Given such pressures, one might expect evolution to have selected for abilities to detect and avoid infection threats. Indeed, existing work suggests people can use certain sensory information to accurately identify whether another person is infected. However, among the five major senses, hearing has been relatively unexamined in this context. Can people identify infectious disease from sounds such as coughs and sneezes? In this paper, we report four studies suggesting the answer to this question might, surprisingly, be no.

Why might infectious disease detection abilities exist? The

Nicholas M. Michalak, Department of Psychology, University of Michigan, Oliver Sng, Department of Psychological Science, University of California-Irvine, Iris M. Wang, Department of Psychology, University of Michigan, Joshua M. Ackerman, Department of Psychology, University of Michigan.

This is an unedited manuscript accepted for publication at Proceedings of the Royal Society B: Biological Sciences. The manuscript will undergo copyediting, typesetting, and review of resultingproof before it is published in its final form..

Correspondence concerning this article should be addressed to Nicholas M. Michalak, 530 Church St, Ann Arbor, MI 48104. Email: nickmm@umich.edu physiological immune system is an evolved mechanism for coping with pathogen threats. However, immune defenses are energetically expensive, and they risk collateral damage. Given these issues, defensive psychological mechanisms that help avoid infection through behavior would complement the physiological immune system. Such mechanisms have been referred to as the behavioral immune system (Ackerman, Hill, \& Murray, 2018; Schaller \& Park, 2011).

Multiple lines of evidence indicate that organisms use sensory cues, specifically those tied to objects and behaviors historically associated with pathogen transmission (e.g., rotting material, feces), to improve pathogen threat detection accuracy. Many species of animals respond to olfactory, visual, tactile, and gustatory cues connoting parasitic or toxic dangers with aversive and sanitation-promoting behaviors (Curtis, 2014; Hart, 1990; Kavaliers \& Colwell, 1995; Sharp, Garnick, Elgar, \& Coulson, 2015). Humans also use a variety of sensory cues to detect pathogen presence, although research with humans is relatively recent and commonly restricted to Western undergraduates. Moreover, human evidence is limited by relatively few studies within any single modality. Reviewing this work, with regard to sight, people were repulsed by fecal-shaped objects, despite explicit knowledge that they are non-infectious (Rozin, Millman, \& Nemeroff, 1986). More generally, stronger disgust reactions emerged when perceivers saw images of body fluid-like stimuli relative to comparable stimuli without such an appearance (e.g., lesions with pus versus a burn scar, Curtis, Aunger, and Rabie 
(2004)). Recent research also found perceivers could distinguish above chance the faces of sick and healthy individuals (Axelsson et al. (2018); but see Kurvers and Wolf (2018)), though visual cues may not be especially helpful for predicting a person's susceptibility to future infection (Cai et al., 2019; Foo, Simmons, \& Rhodes, 2017). With regard to smell, participants judged the odors of healthy individuals injected with lipopolysaccharide (which activates immune function) as less healthy (Olsson et al., 2014) and engaged in prophylactic behaviors when exposed to fecal-smelling chemicals (Tybur, Bryan, Magnan, \& Hooper, 2011). Additionally, Russian participants rated the body odors of those infected with gonorrhea as more unpleasant (Moshkin et al., 2012). Finally, with touch, participants rated objects that were wet and similar to biological material (e.g., a dough mixture, as opposed to cotton rope) as more disgusting and likely to produce illness (Oum, Lieberman, \& Aylward, 2011), whereas inducing disgust in individuals led to enhanced skin sensitivity, ostensibly facilitating avoidance of infectious stimuli (Hunt et al., 2017).

Detection of pathogen threats is important for many species. However, very little work has examined the role of auditory cues in this process. The ability to identify infected individuals by sound seems useful in avoiding infection, especially as it would allow detection from safer distances. Consistent with the potential relevance of sound, people were more likely to wash their hands after hearing belching sounds (Pellegrino, Crandall, \& Seo, 2016). In addition, hearing others cough or sneeze increased perceptions of multiple health threats (Lee, Schwarz, Taubman, \& Hou, 2010). Although these findings require replication, it seems that sounds can elicit pathogen-avoidance responses and behaviors.

But can people accurately detect pathogens through sound? From an error management perspective (Haselton \& Nettle, 2006), people may possess evolved biases that limit accuracy. First, uncertainty afflicts interpersonal pathogen detection, and, second, asymmetric costs in detection errors exist. Mistaking an infectious person as non-infectious and potentially exposing oneself to harmful pathogens is probably costlier than mistaking a non-infectious person as infectious. Thus, people may be biased to judge coughs and sneezes as originating from infected rather than non-infected people. Such a bias-regardless of its potential origins (McKay \& Efferson, 2010)_-would reduce the costlier error (similar to a smoke detector's false-positive bias).

Here, we report a pilot and three studies that (1) test whether people can accurately detect pathogen threats from cough and sneeze sounds, and (2) test potential explanations for any detection abilities. Regarding detection abilities, perceivers may have lay theories about which sound dimensions cue pathogen threat, and these theories may help them reliably diagnose infection. For example, if disgust evolved to promote disease avoidance (Curtis et al., 2004; Tybur, Lieberman,
Kurzban, \& DeScioli, 2013), and people are disgusted by sensory cues that indicate the presence of pathogens, then people may believe that disgusting features of cough and sneeze sounds can appropriately diagnose infection. We tested this both by directing participants to use their disgust reactions to make judgments about sounds that were infectious or noninfectious in origin and by examining associations between disgust ratings and accuracy. Finally, we also tested whether trait-level concerns about infection are associated with detection accuracy.

\section{Research Overview}

An initial pilot study (see supplementary write-up) with 116 participants used similar methods to those described in Study 1 below. After listening to and attempting to identify whether coughs and sneezes were infectious or non-infectious in origin, participants accurately identified $43.79 \%$ of the sounds' origins, $95 \%$ CI [34\%, 54\%], a result not statistically different from chance $(50 \%)$. In our primary studies, we expanded on this finding using a larger stimulus set and more varied measures. For Studies 2 and 3, we preregistered research questions, predictions, sampling plans, exclusion criteria, and analyses on AsPredicted.org or the Open Science Framework. For these and additional analyses, see (Michalak, Sng, Wang, Ackerman, \& Dobbs, 2017, https://osf.io/4c7vr/).

Previewing our findings, we find no evidence that perceivers can accurately detect (above chance) pathogen threat from sounds of coughs and sneezes, even though perceivers are highly certain in their judgments. We do find that the more disgusting that people perceive such sounds, the more likely they are to judge them as originating from infected others. However, attending to this disgust does not appear to improve pathogen threat detection accuracy.

\section{Study 1}

\section{Method}

Participants. Using TurkPrime (Litman, Robinson, \& Abberbock, 2017), we recruited 165 U.S. participants from Amazon's Mechanical Turk system. Participants had completed at least 100 prior MTurk assignments with a 95\% approval rate and were paid $\$ 0.90$. Our final sample comprised 148 participants $\left(M_{\text {age }}=37.02, S D_{\text {age }}=10.73,83\right.$ women $)$, which afforded $80 \%$ power to detect about an $11 \%$ difference from $50 \%$ (Cohen's $h=0.23$; Cohen, 1988). See our supplement for detailed, preregistered exclusions based on hearing issues, medical training, and survey completion (Studies 1-3). Conclusions in all studies are robust to these exclusions.

Materials and Procedure. Sound stimuli featuring coughs and sneezes were extracted from online, U.S.-based videos 
(e.g., YouTube) (see materials supplement). We included different types of sounds to improve ecological validity, though we had no predictions about sound type differences. Targets who generated the infectious sounds self-reported with certainty experiencing sickness with an infectious disease (e.g., cold, flu). Targets who generated the non-infectious sounds responded to benign irritants (e.g., allergies, consumption of powdery spices, cotton swabs). We trimmed videos to 1-2 second audio clips featuring only the target sound. The full stimulus set comprised 20 coughs and 20 sneezes, with half of each sound type being infectious or non-infectious in origin.

After responding positively to a volume check, participants were given instructions about sound identification. Infectious illness was defined as "an illness that can spread between people" and non-infectious was not defined. Sounds were presented in random order.

After each clip, participants answered: (1) "Do you think the sound is from a person with an infectious illness or a person with a non-infectious condition?" (Infectious/Non-infectious), (2) "How certain are you that your above answer is correct?" $(1 / 9=$ Not at all certain/Very certain), and (3) "How clear is the sound in this audio clip (How well could you hear it)?' $(1 / 9=$ Not at all clear/Very clear).

Participants next completed a trait-level index of disease concern, the Perceived Vulnerability to Disease Questionnaire (Duncan, Schaller, \& Park, 2009). To save space, we report models using this scale in our analyses supplement. Finally, participants completed demographic items.

Analyses outline. In all studies, contingent on our dependent variable (e.g., 0/1 = incorrect/correct identification, $1 / 9=$ Not at all certain/Very certain), we used the lme4 package in R (Bates, Mächler, Bolker, \& Walker, 2014) to fit either logistic or linear mixed effects models (deviating from our preregistered Repeated Measures ANOVA analyses). These models account for variability due to Sound Origin (our key variable) while also accounting for sampling variability in participants and sound stimuli. However, the participant factor never accounted for meaningful variance (responses differed more within than between participants), so we excluded it in all reported models (see supplement for all models). We always included Sound Origin (Non-Infectious Origin $=-0.5$, Infectious Origin $=0.5$ ) as a fixed effect. Thus, unless otherwise noted, for all models using accuracy as the dependent measure (reported as percentage correct and log-odds), we included only stimuli intercepts. Importantly, because our diagnostic test was binary, overall accuracy is identical to the Area Under the Receiver Operating Characteristic curve.

In contrast, when we modeled Likert-like dependent measures (e.g., 1/9 = Not at all certain/Very certain), we specified random intercepts for participants and sound clips, and we specified random participant slopes for Sound Origin because we observed participants responding uniquely across the two sound origin categories. We describe additional fixed effect specifications in our Results sections. Because we made no predictions for sound type, we did not include it as a factor in our main analyses (see analysis supplement for models including this factor).

To increase confidence in our conclusions, we repeated the detection analyses using the subset of stimuli containing an explicit infection diagnosis. When comparing judgment accuracy for this subset to every possible non-infectious stimulus set of equal size, statistical conclusions did not differ from those involving the full stimulus set (see supplement). Thus, across studies, we report analyses using all stimuli. In addition, we had 5 research assistants code age, gender, and race/ethnicity of the target individuals featured in the sound stimuli to evaluate as potential confounds. Including these variables in our regressions did not meaningfully affect coefficients nor conclusions, so they are not included in the analyses. Details are provided in the supplement.

\section{Results}

Are people able to accurately identify infectious and noninfectious coughs and sneezes? We find no sufficient evidence that people can. On average, participants correctly identified $45 \%$ of the sounds, consistent with chance $(50 \%), b=-0.22$, $95 \%$ CI $[-0.57,0.13]^{1}$. The upper bound of our confidence interval for overall accuracy translates to $53 \%$, so we can reject overall accuracy above 53\%. Accuracy also did not significantly depend on whether the sounds were truly infectious or non-infectious in origin, 95\% CI [-0.69, 0.71] (see Figure 1). Similar to our pilot study, this low overall accuracy stems from the fact that participants more often classified noninfectious sounds as infectious (55\% False Positive Rate/45\% Specificity) than infectious sounds as infectious (45\% True Positive Rate/Sensitivity).

Despite their accuracy being consistent with chance, perceivers were reasonably certain about their judgments, $M=$ $6.68,95 \%$ CI $[6.48,6.89]$ (above the mid-point of 5). In fact, subjective certainty negatively correlated with accuracy such that for every extra unit more certain participants were about their judgments, their odds of having correctly identified a sound's origin decreased by $5 \%(O R=0.95), b=-0.05,95 \%$ CI $[-0.08,-0.01], z=2.71, p=.007$. However, this association strongly depended on Sound Origin, $b=0.12,95 \%$ CI [0.05, $0.18], z=3.45, p<.001$. Participants who reported higher certainty in judgments of sounds with infectious origins were not significantly more accurate than those who were less certain $(z=0.51, p=.607)$, but participants who reported higher

\footnotetext{
${ }^{1}$ We computed $95 \%$ confidence intervals using the likelihood profile method (Bates et al., 2014).
} 

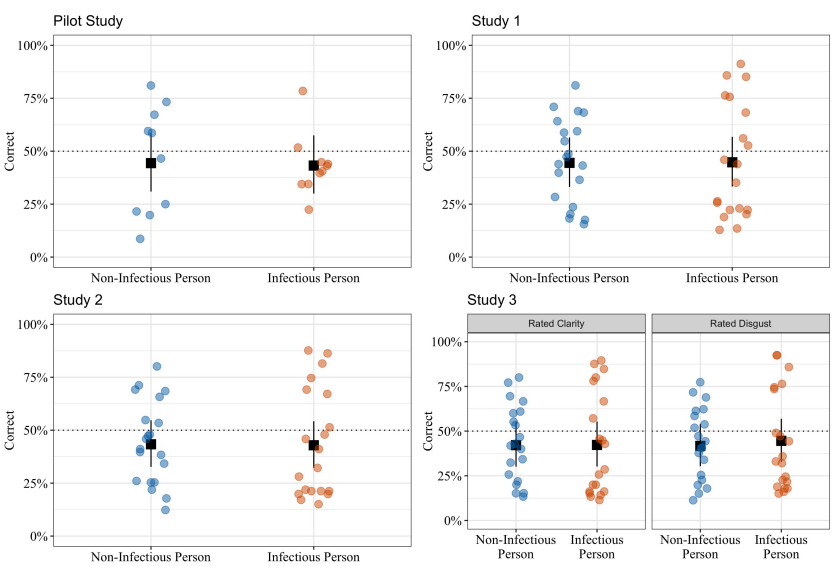

Figure 1. Plots visualize judgment accuracy by Sound Origin across studies (Study 3 depicts separate panels by Sound Rating condition). The dashed lines represent chance levels, and error bars represent profile $95 \%$ confidence intervals based on the standard error of the Sound Origin difference. Points represent average accuracy for stimuli, jittered with random noise to make visible unique accuracy scores.

certainty in their judgments about sounds with non-infectious origins were significantly less accurate than those who were less certain $(z=-4.43, p<.001)$. This might indicate that perceivers are using non-diagnostic auditory cues to infer pathogen threat, a point we return to in the next study.

\section{Study 2}

The results of two initial studies suggest that perceivers may not be able to accurately detect pathogen threats from cough and sneeze sounds. One possibility is that individuals may be relying on misleading auditory information. To test this, in Study 2, we focused on the role of disgust perceptions by instructing participants to rate how disgusting the sound stimuli were before identifying their origin. Given previous work suggesting that pathogen threat cues elicit disgust (Curtis et al., 2004; Oum et al., 2011), attention to disgust might enhance accuracy in this context.

A second issue is that perceivers may have lay beliefs about the natural frequency of infectious sounds in everyday life. In the current paradigm, half of the sound stimuli are infectious in origin, and half are non-infectious (though participants in Study 1 were not told this). If people typically encounter different frequency distributions in their everyday lives, their judgments may reflect those more naturalistic distributions. Therefore, in Study 2, we provided base rates by telling participants that half of the sounds were infectious in origin.

\section{Method}

Participants. One hundred fifty people recruited from MTurk using the same methods in Study 1 participated for $\$ 0.90$. Our final sample comprised 146 participants $\left(M_{\text {age }}=35.14, S D_{\text {age }}\right.$ $=9.73,57$ women), which afforded us $80 \%$ power to detect about a $11 \%$ difference from $50 \%$ (Cohen's $h=0.23$ ).

Materials and Procedure. We used the same stimuli and procedure from Study 1, except for two key differences. First, before hearing the sound clips, participants read: "In total, you will listen to 10 infectious coughs, 10 non-infectious coughs, 10 infectious sneezes, and 10 non-infectious sneezes. These will be randomly ordered, so you will have to judge the type of each sound." Second, before identifying each sound clip as infectious or non-infectious in origin, participants were instructed to rate "How disgusting do you find this sound?" (1/9 = Not at all disgusting/Very disgusting).

\section{Results}

Given perceiver knowledge of sound origin base rates and prior ratings of disgust, were participants able to accurately identify infectious and non-infectious cough and sneeze sounds? Still, we found no sufficient evidence for this ability. On average, participants correctly identified $42 \%$ of the sounds as either infectious or not, again not significantly different from chance ${ }^{2}, b=-0.28,95 \%$ CI $[-0.61,0.05]$. The upper bound of the confidence interval for overall accuracy translates to $51 \%$, so we can reject overall accuracy above $51 \%$. Also like in Study 1, accuracy did not significantly depend on whether the sounds were truly infectious or non-infectious in origin, 95\% CI [-0.68, 0.64], $p=.954$ (Figure 1). Similar to Study 1 , low accuracy stems from the fact that participants more often classified non-infectious sounds as infectious $(56 \%$ False Positive Rate/44\% Specificity) than infectious sounds as infectious (44\% True Positive Rate/Sensitivity).

Were disgust ratings associated with judgment accuracy? We find no sufficient evidence for an average association between disgust ratings and accuracy, 95\% CI [-0.01, 0.05]. However, this association strongly depended on Sound Origin, $b=$ $0.75,95 \%$ CI $[0.69,0.81], z=25.11, p<.001$. For sounds with infectious origins, the more disgusting that participants perceived those sounds, the more accurately they identified them (i.e., judging them correctly as having an infectious origin). For sounds with non-infectious origins, the more disgusting that participants perceived those sounds, the less accurately they identified them (i.e., judging them incorrectly as having an infectious origin). These patterns are consistent

\footnotetext{
${ }^{2}$ Here and across studies, overall accuracy falls below 50\%. Adjusting for sound type (cough or sneeze), target demographics, and sound disgustingness did not substantively change this pattern.
} 
with the hypothesis that disgust response is used as an index for pathogen presence: If they perceive a person's cough or sneeze as disgusting, they are more likely to judge it as having an infectious origin.

As in Study 1, participants were reasonably certain about their judgments, $M=6.08$, 95\% CI [5.80, 6.35] (above the midpoint of 5). For every additional unit of certainty, the odds of their accurately judging the sound's origin decreased by $4 \%$ $(O R=0.96), b=-0.04,95 \%$ CI $[-0.07,-0.01], z=2.89, p=$ .004 . Unlike in Study 1, we found no sufficient evidence this association depended on Sound Origin, 95\% CI [-0.03, 0.08].

Comparing Study 2 to Study 1, it seems that focusing on how disgusting cough and sneeze sounds are perceived does not significantly improve judgment accuracy for those sounds' origin. Perceivers did rate sounds they thought were disgusting as more likely to be infectious in origin. This may reflect perceivers' lay theories that more disgusting sounds are more likely to be infectious. However, Study 2 lacked a control condition, which would allow us to directly test whether orienting participants to how disgusting the sounds were-compared to another subjective quality-improves accuracy. In Study 3 , we randomly assigned participants to rate sounds on either disgust or clarity before making origin judgments.

\section{Study 3}

\section{Method}

Participants. Two hundred twenty-four people recruited from MTurk using the methods from prior studies participated for $\$ 1.00$. Our final sample comprised 211 participants $\left(M_{a g e}\right.$ $=37.01, S D_{\text {age }}=12.24,108$ women), which afforded us $80 \%$ power to detect about a $10 \%$ difference from $50 \%$ (Cohen's $h$ $=0.19)$.

Materials and Procedure. Study 3 followed the same procedures as Study 2 except we randomly assigned participants to rate disgust $(n=106)$ or clarity $(n=105)$ for each sound before judging its origins. Participants read: "Research has shown that people are better at identifying the origin of sounds like the ones you'll hear when they pay attention to the sounds' (auditory clarity/disgusting quality). Therefore, when you attempt to identify each sound, pay special attention to (how clear the sound is/how disgusting the sound is) before making your decision." For each sound clip, participants responded to the question "How [clear/disgusting] do you find this sound?" $(1 / 9=$ Not at all [clear/disgusting]/Very [clear/disgusting]).

\section{Results}

Are people able to accurately identify the origins of infectious and non-infectious cough and sneeze sounds when explicitly attending to how clear or disgusting the sounds are? On average, participants accurately identified sound origin $43 \%$ of the time, not significantly different from chance, $b=-0.30,95 \%$ CI $[-0.66,0.07]$. The upper bound of the confidence interval for overall accuracy translates to $52 \%$, so we can reject overall accuracy above 52\%. Also like in Studies 1-2, accuracy did not significantly depend on whether the sounds were truly infectious or non-infectious in origin, 95\% CI [-0.67, 0.79] (Figure 1). Similar to Studies 1 and 2, low overall accuracy stems from participants more often classifying non-infectious sounds as infectious (57\% and 56\% False Positive Rates/43\% and $44 \%$ Specificity for Disgust and Clarity Conditions) than infectious sounds as infectious (45\% and 43\% True Positive Rates/Sensitivities for Disgust and Clarity Conditions).

Were clarity and disgust ratings associated with accuracy? We found no significant average association, but the association between sound rating and accuracy significantly depended on both Sound Origin and Rating Condition (a 3-way interaction) (Figure 2), $b=1.02,95 \%$ CI $[0.91,1.14], z=17.88, p<.001$. In the Disgust Rating Condition, the more disgusting participants perceived sounds with infectious origins, the more accurately they identified them $(z=17.98, p<.001)$, but the more disgusting participants perceived sounds with noninfectious origins, the less accurately they identified them $(z=-18.14, p<.001)$. This is similar to the pattern found in Study 2. In contrast, we found no sufficient evidence for associations between perceived clarity and accuracy $(z s=$ 0.07 and -0.25 and $p s=.947$ and .800$)$.

As in Studies 1-2, participants were reasonably certain about their judgments, $M=6.35,95 \%$ CI $[6.14,6.56]$ (above the mid-point of 5). For every additional unit more certain participants rated their judgment, the odds of their accurately judging sound origin decreased by $4 \%(O R=0.96), b=-0.04$, $95 \%$ CI $[-0.065,-0.01], z=2.97, p=.003$.

\section{General Discussion}

Can perceivers detect pathogen threats from cough and sneeze sounds? Given our hypothesis that this capacity would be adaptive for limiting pathogenic exposure, and existing work indicating similar capacities with other sensory modalities, we predicted that people would be able to accurately detect pathogen threat using auditory cues. Across four studies, we found no support for this prediction. Moreover, there was no evidence that accuracy improved when participants knew the true number of infectious sounds in advance or when participants focused on how clear or disgusting they perceived the sounds. Despite this poor overall accuracy, perceivers consistently reported reasonable certainty in their judgments.

Poor accuracy notwithstanding, we identified one subjective dimension used to identify the origin of sounds. In Studies 2 

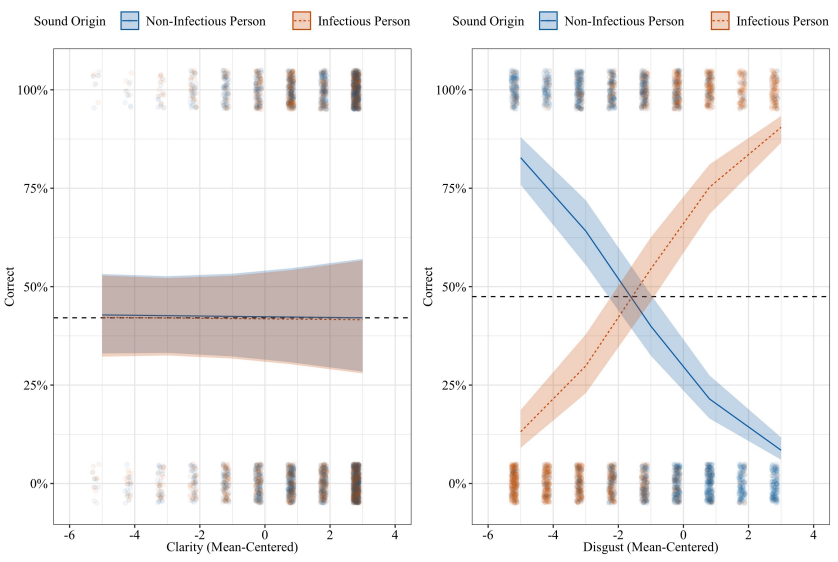

Figure 2. Plots visualize the relation between sound rating (clarity or disgust) and judgment accuracy by sound origin (infectious or non-infectious targets). Higher ratings of disgust correlated positively with accuracy for infectious sounds but negatively with accuracy for non-infectious sounds (clarity ratings had no significant effect). Dashed lines represent adjusted average accuracy for each condition. Small points represent raw judgments "jittered" with random noise to make visible unique judgments. Ribbons around the lines represent 95\% confidence intervals based on the three-way interaction (see effects package in R, Fox \& Hong, 2009)

and 3 , the more disgusting that participants rated sounds, the more likely they were to judge a sound as infectious in origin, regardless of whether it truly was. This was not the case for another subjective dimension-sound clarity. It seems that perceivers possess the lay theory that what disgusts them is likely to represent a disease threat, potentially leading them to exhibit biases to avoid interactions with others who make disgusting but non-infectious noises.

Given these findings, should we conclude that people possess no auditory disease detection mechanism? Perhaps coughs and sneezes are such strong and consistent physiological reflexes that any variety of causes, infectious or not, will produce very similar sounds. If so, the necessary sound variation would not have been available to natural selection processes (or perceivers). Such sound variation could be very limited, but recent work suggests that this variation is available (e.g., to statistical learning algorithms; Porter et al., 2019). However, human hearing mechanisms may not be able to use it reliably, even with clinical training (e.g., Smith, Ashurst, Jack, Woodcock, \& Earis, 2006). Another possibility is that people integrate available sickness sound information with other sensory information (e.g., Regenbogen et al., 2017) rather than perceiving it in isolation. Accuracy may improve when people hear sounds alongside other sensory cues (e.g., seeing someone sneeze, hearing someone talk with a hoarse voice). Of course, the same is true for other sensory modalities, including ones that have been tested in isolation and found to predict accurate identification (e.g., Axelsson et al., 2018). A final possibility is that our stimuli may not represent the relevant range of sounds that perceivers encounter in natural settings. We tried to address this by using a range of sound types (i.e., coughs and sneezes), but our set may have nonetheless been limited in type, quality, and breadth of eliciting conditions.

People certainly attend and react to auditory information in contexts related to infectious disease threats (Lee et al., 2010; Pellegrino et al., 2016). Yet, our data suggest they are poorly able to distinguish infectious cough and sneeze sounds from benign ones. From an error management perspective, biases to presume that coughs and sneezes indicate pathogen presence could be functional if sound origin is uncertain and the costs of mistaking benign coughs and sneezes for infectious ones are lower than the costs of mistaking infectious ones as benign. However, this also does not appear to fully explain our results. Individual differences in self-perceptions of vulnerability to disease were not significantly associated in a consistent manner with inferences of infectious threat (see supplement), as this perspective might predict.

Work on the psychological mechanisms that manage infectious disease threat has recently flourished, coupled with advances in our understanding of the selection pressures that pathogens have exerted on human evolution. Despite this growing literature, relatively little research has focused on the manner by which people accurately identify pathogen threats. The work presented here on auditory detection expands on this emerging literature. Relevant information about sickness is carried through sound, and perceivers believe this information to be both useful and relatively safe (Ackerman, Merrell, \& Choi, 2020), making it useful to know whether auditory information is interpreted accurately. Adaptive explanations also exist to predict either accurate or biased perception of infection threats through sounds. Though the nascent state of the literature makes this investigation largely exploratory, the current work speaks to these alternative hypotheses and advances future theorizing about pathogen threat processing.

In conclusion, we find no evidence that perceivers can reliably detect pathogen threats from cough and sneeze sounds, even though they are reasonably certain they can. Perceivers seem to use the disgustingness of these sounds to infer pathogen threat but paying attention to their disgust does not significantly improve accuracy. Thus, the next time you hear someone cough or sneeze, perhaps leave the diagnosis to the doctor. 


\section{References}

Ackerman, J. M., Hill, S. E., \& Murray, D. R. (2018). The behavioral immune system: Current concerns and future directions. Social and Personality Psychology Compass, 12(2), e12371. https://doi.org/10.1111/ spc3.12371

Ackerman, J. M., Merrell, W. M., \& Choi, S. (2020). What people believe about detecting infectious disease using the senses. Manuscript Submitted for Review.

Axelsson, J., Sundelin, T., Olsson, M. J., Sorjonen, K., Axelsson, C., Lasselin, J., \& Lekander, M. (2018). Identification of acutely sick people and facial cues of sickness. Proceedings of the Royal Society B: Biological Sciences, 285(1870), 20172430.

Bates, D., Mächler, M., Bolker, B., \& Walker, S. (2014). Fitting linear mixed-effects models using lme4. Journal of Statistical Software, 67(1), 1-48. https: //doi.org/10.18637/jss.v067.i01

Cai, Z., Hahn, A. C., Zhang, W., Holzleitner, I. J., Lee, A. J., DeBruine, L. M., \& Jones, B. C. (2019). No evidence that facial attractiveness, femininity, averageness, or coloration are cues to susceptibility to infectious illnesses in a university sample of young adult women. Evolution and Human Behavior, 40(2), 156159.

Cohen, J. (1988). Statistical power analysis for the behavioral sciences (2nd ed.). Hillsdale,NJ: Lawrence Erlbaum.

Curtis, V. A. (2014). Infection-avoidance behaviour in humans and other animals. Trends in Immunology, 35(10), 457-464.

Curtis, V., Aunger, R., \& Rabie, T. (2004). Evidence that disgust evolved to protect from risk of disease. Proceedings of the Royal Society of London. Series B: Biological Sciences, 271(suppl_4), S131-S133.

Duncan, L. A., Schaller, M., \& Park, J. H. (2009). Perceived vulnerability to disease: Development and validation of a 15-item self-report instrument. Personality and Individual Differences, 47(6), 541-546. https://doi.org/10.1016/j.paid.2009.05.001

Foo, Y. Z., Simmons, L. W., \& Rhodes, G. (2017). Predictors of facial attractiveness and health in humans. Scientific Reports, 7, 39731.

Fox, J., \& Hong, J. (2009). Effect displays in R for multinomial and proportional-odds logit models: Extensions to the effects package. Journal of Statistical Software, 32(1), 1-24.
Hart, B. L. (1990). Behavioral adaptations to pathogens and parasites: Five strategies. Neuroscience $\mathcal{E}$ Biobehavioral Reviews, 14(3), 273-294.

Haselton, M. G., \& Nettle, D. (2006). The paranoid optimist: An integrative evolutionary model of cognitive biases. Personality and Social Psychology Review, 10(1), 47-66.

Hunt, D. F., Cannell, G., Davenhill, N. A., Horsford, S. A., Fleischman, D. S., \& Park, J. H. (2017). Making your skin crawl: The role of tactile sensitivity in disease avoidance. Biological Psychology, 127, 4045.

Karlsson, E. K., Kwiatkowski, D. P., \& Sabeti, P. C. (2014). Natural selection and infectious disease in human populations. Nature Reviews Genetics, 15(6), 379.

Kavaliers, M., \& Colwell, D. D. (1995). Discrimination by female mice between the odours of parasitized and non-parasitized males. Proceedings of the Royal Society of London. Series B: Biological Sciences, 261(1360), 31-35.

Kurvers, R. H., \& Wolf, M. (2018). Identification of acutely sick people: Individual differences and social information use. Proceedings of the Royal Society B, 285(1889), 20181274.

Lee, S. W., Schwarz, N., Taubman, D., \& Hou, M. (2010). Sneezing in times of a flu pandemic: Public sneezing increases perception of unrelated risks and shifts preferences for federal spending. Psychological Science, 21(3), 375-377.

Litman, L., Robinson, J., \& Abberbock, T. (2017). TurkPrime. Com: A versatile crowdsourcing data acquisition platform for the behavioral sciences. Behavior Research Methods, 49(2), 433-442. https://doi.org/10. 3758/s13428-016-0727-z

McKay, R., \& Efferson, C. (2010). The subtleties of error management. Evolution and Human Behavior, 31(5), 309-319.

Michalak, N. M., Sng, O., Wang, I., Ackerman, J., \& Dobbs, S. (2017). Sounds of sickness: Can people identify infectious disease using sounds of coughs and sneezes? https://doi.org/10.17605/OSF.IO/4C7VR

Moshkin, M., Litvinova, N., Litvinova, E. A., Bedareva, A., Lutsyuk, A., \& Gerlinskaya, L. (2012). Scent recognition of infected status in humans. The Journal of Sexual Medicine, 9(12), 3211-3218.

Olsson, M. J., Lundström, J. N., Kimball, B. A., Gordon, A. R., Karshikoff, B., Hosseini, N., ... Lekander, M. (2014). The scent of disease: Human 
body odor contains an early chemosensory cue of sickness. Psychological Science, 25(3), 817-823. https://doi.org/10.1177/0956797613515681

Organization, W. H. (2018). Global Health Estimates 2016: Deaths by Cause, Age, Sex, by Country and by Region, 2000-2016. Geneva: World Health Organization. Retrieved from https://www.who.int/healthinfo/global_burden_ disease/GHE2016_DALYs-2016-country.xls?ua=1

Oum, R. E., Lieberman, D., \& Aylward, A. (2011). A feel for disgust: Tactile cues to pathogen presence. Cognition and Emotion, 25(4), 717-725.

Pellegrino, R., Crandall, P. G., \& Seo, H.-S. (2016). Using olfaction and unpleasant reminders to reduce the intention-behavior gap in hand washing. Scientific Reports, 6, 18890.

Porter, P., Abeyratne, U., Swarnkar, V., Tan, J., Ng, T.-w., Brisbane, J. M., ... Della, P. (2019). A prospective multicentre study testing the diagnostic accuracy of an automated cough sound centred analytic system for the identification of common respiratory disorders in children. Respiratory Research, 20(1), 81. https://doi.org/10.1186/s12931-019-1046-6

Rozin, P., Millman, L., \& Nemeroff, C. (1986). Operation of the laws of sympathetic magic in disgust and other domains. Journal of Personality and Social Psychology, 50(4), 703.

Schaller, M., \& Park, J. H. (2011). The behavioral immune system (and why it matters). Current Directions in Psychological Science, 20(2), 99-103. https://doi.org/10.1177/0963721411402596

Sharp, J. G., Garnick, S., Elgar, M. A., \& Coulson, G. (2015). Parasite and predator risk assessment: Nuanced use of olfactory cues. Proceedings of the Royal Society B: Biological Sciences, 282(1817), 20151941.

Smith, J. A., Ashurst, H. L., Jack, S., Woodcock, A. A., \& Earis, J. E. (2006). The description of cough sounds by healthcare professionals. Cough (London, England), 2, 1. https://doi.org/10.1186/1745-9974-2-1

Tybur, J. M., Bryan, A. D., Magnan, R. E., \& Hooper, A. E. C. (2011). Smells like safe sex: Olfactory pathogen primes increase intentions to use condoms. Psychological Science, 22(4), 478-480.

Tybur, J. M., Lieberman, D., Kurzban, R., \& DeScioli, P. (2013). Disgust: Evolved function and structure. Psychological Review, 120(1), 65-84. 